\title{
Case Study on Large Ingots Cracking Failure
}

${ }^{1} S$ M Nazmuz Sakib (Orchid- https://orcid.org/0000-0001-9310-3014)

${ }^{1}$ Graduate of BSc in Business Studies

School of Business And Trade

Pilatusstrasse 6003, 6003 Luzern, Switzerland

sakibpedia@gmail.com

${ }^{1}$ Student of Department of Law

Dhaka International University

House \# 4, Road \# 1, Block - F, Dhaka 1213

sakibpedia@students.diu.ac 


\begin{abstract}
Use of Metal and its alloys have become the need of this world. high strength pipes and equipment are required for the extraction of bituminous oil from soil. For this High strength Low Alloy Steel (Micro-alloyed steels) can be used. HSLA required large size ingots for its manufacturing therefore in recent years, large size ingots demand is increased. Large size ingots are subjected to cracking while manufacturing during open die forging process. Optical Scanning Electron Microscopies and Energy dispersion Spectroscopy techniques are carried out for the investigation of root causes of the cracking during forging of large size ingots. Microstructure of large size ingots sample are reviewed at different locations and grain boundaries. Results of these chemical techniques shows the enrichment of chromium and oxygen content at the boundaries which clearly differentiated a cracked boundary and non-cracked locations. This writing comprises the literature study, test procedures and analyzing the results to review that abnormal grain growth was the cause of cracking of large size ingots during forging process. The report also reviewed, alternate heat treatments and possible solution.
\end{abstract}




\section{CASE STUDY | INVESTIGATION OF LARGE SIZE INGOTS CRACKING}

\section{FAILURE 1. Introduction}

For past few decades, Industries are revolutionized in metallurgical sector. Use of metal and its alloys have become the need of the industries. For the manufacturing of pipes and equipment to dig out soil for the extraction of crude bituminous oil (Petrochemical Industry), high mechanical properties material is required. These mechanical properties are toughness, hardness and wear resistance of the material. Metal itself cannot compete these properties. Therefore, Metal alloys are formed to achieve desired properties.

Commonly used alloys are ferrite alloys. Ferrite alloys have low thermal expansion coefficient whereas high thermal conductivity. The ferrite carbon steel alloys' mechanical properties can vary due to change in their composition and microstructure which depends upon/ are the function of grain size, precipitates, secondary phases etc. At high temperature, ferritic steel creep properties are very poor because of the Abnormal Grain Growth. This Grain growth is normal at low temperatures but abnormal at high temperatures. This abnormal growth cause reduction in desired strength and properties.

(Bhadeshia and Honeycombe, 2017, Llewellyn and Hudd, 1998, Totten, 2006, Totten

et al., 2004, Mandal, 2015)

High strength Low Alloy-HSLA (Micro-alloyed steel) can be used for the extraction process as it possesses high mechanical properties. High Strength Low Alloy (HSLA) Steel also referred as Micro-alloyed steel. HSLA has high mechanical properties that are achieved by using medium carbon low alloyed steel with the addition of some other elements by $0.1 \%$ weight. These other elements may include Titanium, Niobium or Vanadium with $2 \%$ by weight of Nickel and Chromium. HSLA steel has high resistance against corrosion as compared to the Conventional/Old Steels (Carbon).

HSLA Steels are produced by casting of ingots. These ingots are manufactured through several open die forging passes at temperature around $1000^{\circ} \mathrm{C}$ which improves hardness of the material. After forging, material is air cooled and tempered which form the bainitic microstructure through ingot thickness. A complex structure of the material is formed after forging, quenching, tempering and normalizing in which carbides, bainite and martensite are present. 
(KONSTRUKCIJSKA, 2011, Wang, 2017, Manohar, 1997)

\subsection{Case Study Problem Statement}

Manufacturing of Large size ingots have been increased not only for petrochemical industry but also for various industries. Critical point in manufacturing process of ingots is the forging process at high temperature which is producing cracking. This cracking phenomenon is the point of discussion in this case study report. For the extraction process of bituminous oil, large size ingots of HSLA have to be prepared. But these ingots showing cracking mechanism during the forging process.

Metals and its alloys are subjected to very high temperature while its manufacturing during various processes. Not only in manufacturing phase, but also in service life, they are subjected to a very high temperature. The Case study spotted light on Abnormal Grain Growth Failure. This failure occurs with the metals working or exposing to a very high temperatures either during manufacturing phase or the service life (Nuclear Reactor cladding example). Type of Metal selection depends upon the mode of serviceability expected. Like if nuclear radiator cladding to be made then metal must be having high temperature and radiations resistive property. Anyhow a metal has to face high temperature while in manufacturing phase.

In current case study, HSLA- Metal Alloy is used for the extraction process. Ingots of HSLA experienced cracking at high temperature during forging process. The cracking is expected due to the Abnormal Grain Growth, Precipitation or segregation of the Metal-Alloy Particles. This writing is done to understand the real cause of the cracking in ingots at high temperature on experimentation and literature basis.

(Bitterlin et al., 2016)

\section{Literature}

\subsection{Grain Growth in Metal Alloys}

When a poly-crystalline metal is annealed, average area of the grain particle increases and causing the grain boundaries to decrease. This Phenomenon referred as the grain growth process. 


\subsubsection{Effect of Grain Growth on Properties of Metal}

Grain growth directly affects the physical and mechanical properties of the metal. Increase in grain size may cause the followings:

Decreases hysteresis and eddy current losses

Reduction in yield strength

Decreases formability

Decreases creep resistance and increases creep strength

Decreases Toughness
Decreases hinderance to stress corrosion

Decreases resistance to temper embrittlement Decreases fatigue strength Increases hardenability Affect optical transmission and material conductance

\subsubsection{Grain Growth Mechanism}

Following are points related to the mechanism of grain growth:

Grain growth occur due to migration of grain boundaries instead neighboring grains.

Grain Boundary migration is jerky or discontinuous process, changes with time and boundary to boundary.

A grain grow into neighboring grain and consume by other neighbor.

$\square$ Consumption rate is fast when a grain is about to disappear in its neighbor.

$\square$ Grains with angle $<120^{\circ}$ degree will be unstable and $\geq 120^{\circ}$ will be stable.

\subsubsection{Types of Grain Growth}

Grain Growth can be normal or abnormal. Grain growth accompanied with uniformity, stability, scaling and log normality, such growth is normal and vice versa is abnormal. 


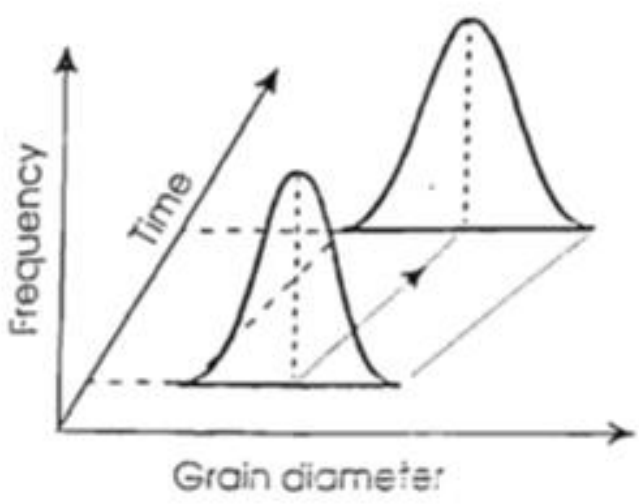

(a)

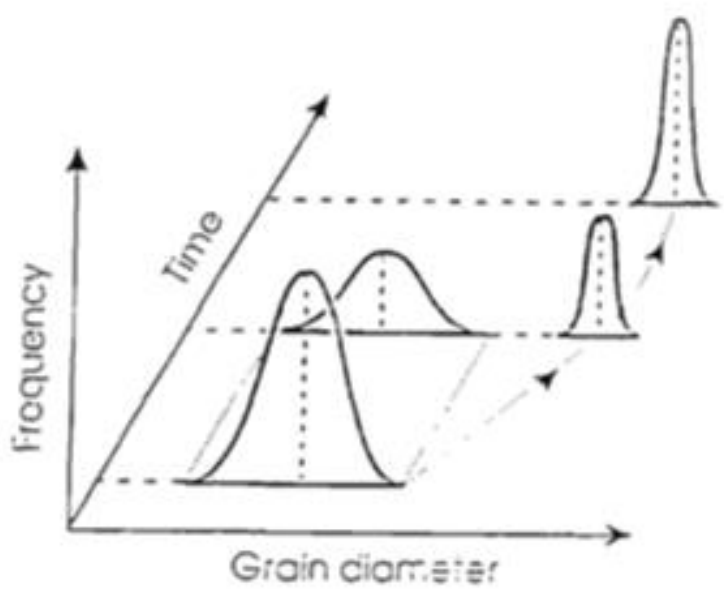

(E)

Figure 1: Schematic diagram for Normal (a) and abnormal (b) grain growth

For normal growth, it is very obvious, grain boundary must be straight and boundary tensions must be equals to 120 degrees. Abnormal is vice versa.

Abnormal grain growth also referred as the secondary recrystallization which accompanied with the consumption of small grains which continue until its microstructure size become large $(\mathrm{mm})$. Some observations regarding abnormal grain growth are as follows:

These grains are the part of initial structure not freshly nucleated in later phase.

$\square$ Its growth controlled by nucleation and have sufficient incubation period.

$\square$ Gain particle size is greater than average.

$\square$ Orientation texture of the abnormal portion is different from the others.

$\square$ It occur when metal passes Grain Coarsening Temperature.

\subsubsection{Conditions of Grain Growth}

Largest grain must be $>1.5$ times the average size.

Grains with average size must be pinned.

$\square$ Log-normal distribution is wide initially.

Pinning effect: the grains which entrap by the larger grains and not able to move. Such a phenomena referred as pinning effect. 


\subsubsection{Grain Coarsening Temperature}

The temperature above which abnormal grain growth become dominant is referred as grain coarse temperature. GCT varies material to material. Knowledge about GCT of specific material is important before subjecting material to hot rolling. Because in first step of Hot rolling (Re-heating Temperature) the amount of microalloying steel and grain size in solution is controlled. This grain size and micro-alloy concentration in real, control the properties like precipitation, grain size growth, phase transformation and re-crystallization of the material. If the reheating temperature is equal to or greater than GCT then micro-alloy will not be able to precipitate as desired and mechanical properties will be compromised.

\subsection{Thermomechanical Processing (TMP) in metal alloys}

The process is defined as Hot deformation schedule, consisting of series of processes (Rolling, Forging, Extrusion etc.) to attain a desired microstructure of the alloy before its final casting/ transformation.

\subsection{Conventional Hot Rolling (CHR)}

The process followed by the reheating of alloy to austenite, soaking and continuous rolling to desired thickness which is subsequently followed by air cooling to room temperature. In First two stages of Conventional Controlled Rolling (CCR) is similar as CHR whereas rolling further has two stages i.e. roughing (Where deformation carried out above critical or Noncrystallization temperature) and finishing (Deformations occur below non-Crystallization Temperature). Between Roughing and finishing, there is enough time delay to fall the rolling stock temperature below Non-Crystallization temperature which causes the flattering of grains as grains are unable re-crystallized. 


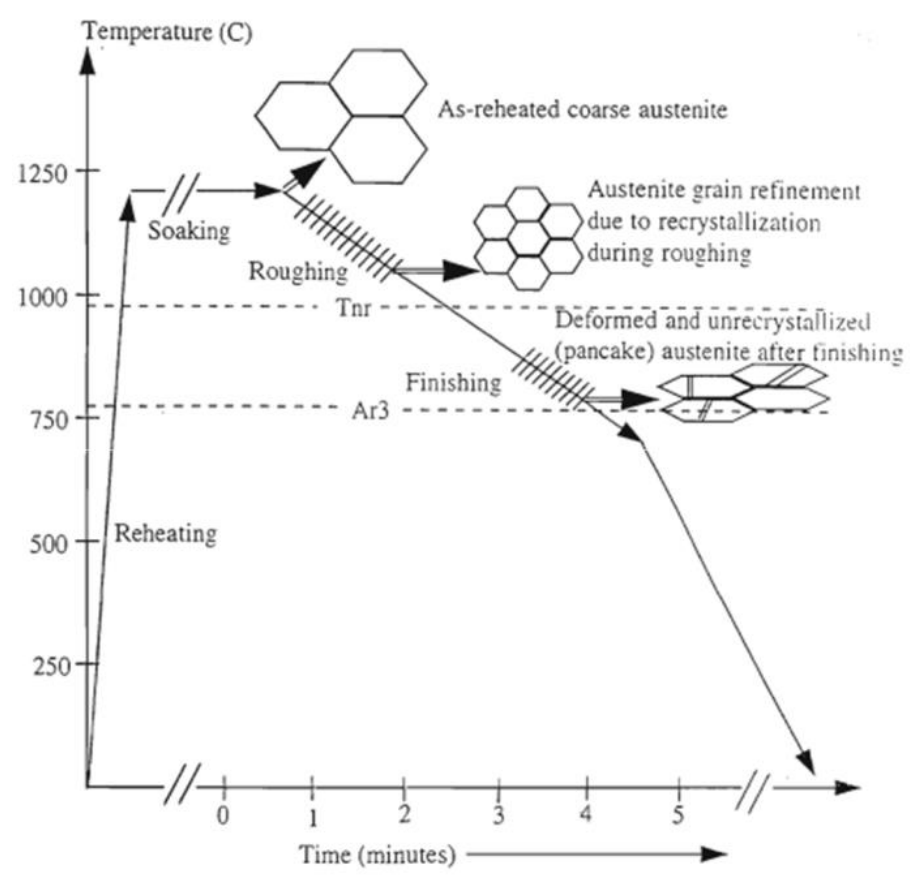

Figure 2: Temperature Profile for CCR

\subsection{Micro-Alloyed Steel}

High Strength Low Carbon Steel Alloy (HSLA) is called as Micro-Alloyed steel. HSLA steel have been successful in recent years due to its strength properties. Its mechanical properties depend upon the morphology, Grain Refinement, carbon nitrides solubility and precipitation, hardening.

\subsubsection{Micro-alloy Composition}

For this, steel is alloyed by the Micro-alloyed elements with the allowable defined \%ages:

Table 1: Composition of Steel Alloy:

\begin{tabular}{|c|c|}
\hline Element & \%age Range \\
\hline $\mathrm{C}$ & $0.06-0.12$ \\
\hline $\mathrm{Mn}$ & Upto 1-2 \\
\hline $\mathrm{Mo}$ & Upto 1 \\
\hline $\mathrm{Ni}$ & Upto 3 \\
\hline $\mathrm{Cr}$ & Upto 1 \\
\hline $\mathrm{Nb}$ & $0.03-1$ \\
\hline $\mathrm{Ti}$ & $0.01-0.05$ \\
\hline $\mathrm{B}$ & $0.0018-0.006$ \\
\hline $\mathrm{V}$ & $0.06-0.15$ \\
\hline
\end{tabular}


$\mathrm{Al}$

0.03-0.08

Amounts of these elements are added approximately $0.1 \%$. HSLA has wide applications in gas and oil pipelines, offshore drilling platforms, structural engineering, ship building, mining equipment, automotive parts etc. it is prior over conventional steels due to improvement in mechanical properties.

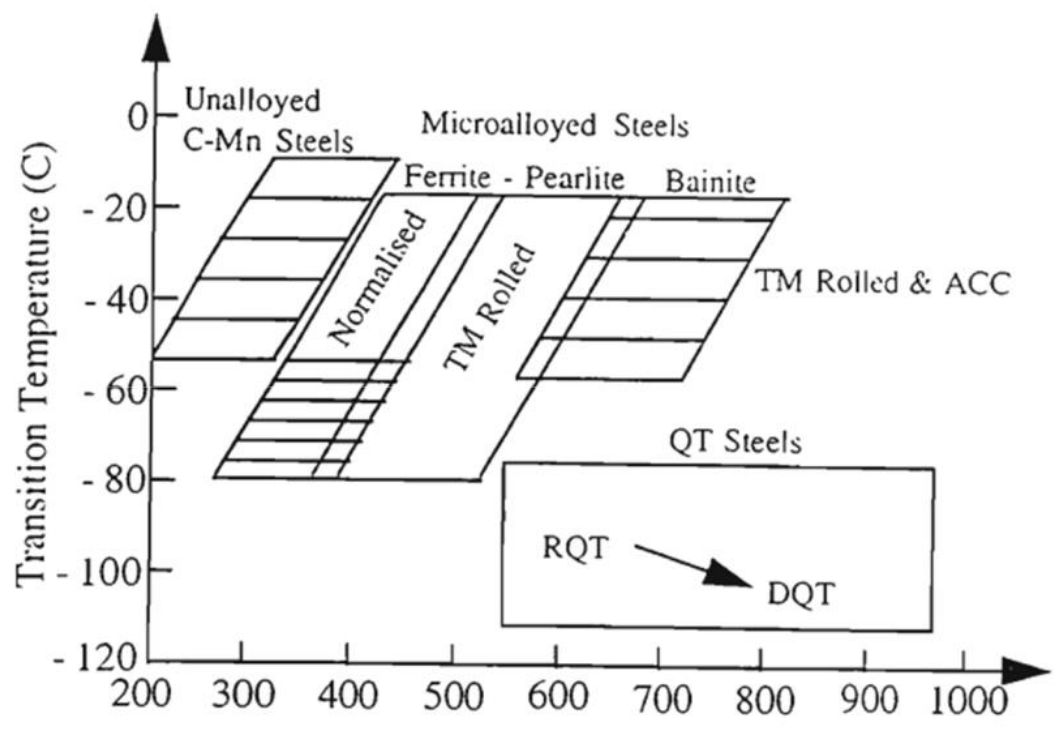

Yield Strength $(\mathrm{MPa})$

Figure 3: Comparison of strength/toughness between micro-alloyed steel and TMP conventional C-Mn steel

Figure clearly states that combination of Micro-alloyed-TMP has almost 4 times more strength as compared to Conventional Steel due to grain refinement, dislocation strengthening, precipitation hardening, solid solution hardening and controlling of microstructure.

\subsubsection{Effect of Micro-Alloyed elements on Mechanical Properties}

Cobalt: it improves precipitation hardening kinetics and martensite formation.

Vanadium: strong carbide and nitride former.

Nickel: it is a hardenability agent like carbon and reduces toughness.

Chromium: it is a hardenability agent, solid solution strengthener, carbides former and mainly make steel as corrosion resistive. 
Niobium: it retards the recrystallization by precipitating the NbCN. It increases the noncrystallization temperature. It increases ferrite strength by precipitating hardening.

Titanium: It improves grain size by precipitating as TiN. It has same function as $\mathrm{Nb}$ but less effective. It hinder $\mathrm{MnS}$ precipitation as it has more affinity for $\mathrm{S}$ to form Carbosulfides.

Nitrogen: along with Ti it produces optimum properties when $\mathrm{Ti}: \mathrm{N}<3.42$.

Silicon: it enhances the yield and tensile strengths without disturbing Elongation (\%) and yield ratio. Above $1 \%$ it promotes Bainitic and Martensitic transformations. High amount of Si, tights TiN instead $\mathrm{TiO}$ which improves grain refinement and decreases hardenability.

Molybdenum: it increases yield and tensile strength of ferrite and above $0.4 \%$ content it affects the toughness. It is also solid solution strengthener and carbide former.

Manganese: it is deoxidizer, hardenability agent and it improves yielding and tensile strengths. In addition, it stops NbCN precipitation with the help of Mo.

Aluminum: Strong nitride former to form AlN to pin grains.

Carbon: Carbon increases hardenability and strengthened the solid solution. It decreases toughness and forms cracks.

\subsubsection{Types of Micro-Alloy Steel}

i. Weathering Steels

ii. Ferrite Pearlite Steels

iii. As-rolled pearlite Steels

iv. Acicular Ferrite Steels v. Dual Phase Steels

vi. Inclusion Shape Controlled Steels

\subsubsection{Strengthening Philosophy in Micro-Alloy Steels}

There are following mechanisms in this regard:

In Dislocation Strengthening, dislocation movement is hindered due to presence of obstacles at boundaries, it may happen that saturation of dislocation occur in solution.

Grain size strengthening is done by grain refinement but it may reduce toughness. 
Solid solution strengthening is done due to presence of solute atom in middle of lattice which hinder the dislocation movement. Solubility limitation is drawback here.

Precipitation strengthening is done by the second phase particles formation which hinder dislocation movement. It affects the grain size and properties.

In Texture strengthening, texture is improved to hinder dislocation movement to impart strength but achieving texture is difficult in steel.

Phase transformation strengthening create new phases which hinder dislocation movement.

Among all mechanisms, grain refinement is preferred mechanism to achieve strength.

\subsubsection{Recrystallization behavior in Micro-alloyed Steel}

Thermomechanical processing accompanied with reheating and soaking processes which involve roughness (High temperature deformations). Roughing process target to achieve fine and uniform grain size by reducing thickness through repeated re-crystallization during rolling process. that uniform and fine grain size. Statics recrystallization are delayed in micro-alloyed steel as compared to conventional C-Mn steel to achieve recrystallization with sufficient deformations before next pass. Abnormal Grain (Partial Re-crystallization) may cause due to light reductions during roughing process.

\subsubsection{Precipitation Phenomenon in Micro-Alloyed Steel}

Micro-Alloy Precipitation in TMP is critical as it account for the mechanical properties. During reheating and soaking process, Grain Coarsening of micro-alloyed depends upon the thermal Stability of the precipitate. Recrystallization kinetics is strongly affected by the strain induced precipitation (Growth rate of grains is directly proportional with product of solute supersaturation and inversely relation with diffusion, now if diffusion decreases under cooling, growth of grains will decreases which reduces the precipitation amount) during the deformation process. Precipitation before and after Transformation phase is important as before it affects transformation kinetics and afterwards it increases ferrite strength through precipitation hardening. During each stage of thermomechanical processing, amount of precipitate will conclude the grain size definition and so accordingly the mechanical properties. 


\subsubsection{Relation between Precipitation and Recrystallization in Micro-alloyed steel}

During Thermomechanical Processing of micro-alloyed steel, interaction between precipitation and recrystallization is represented by the RPTT-diagram proposed by Hansen and given as following:

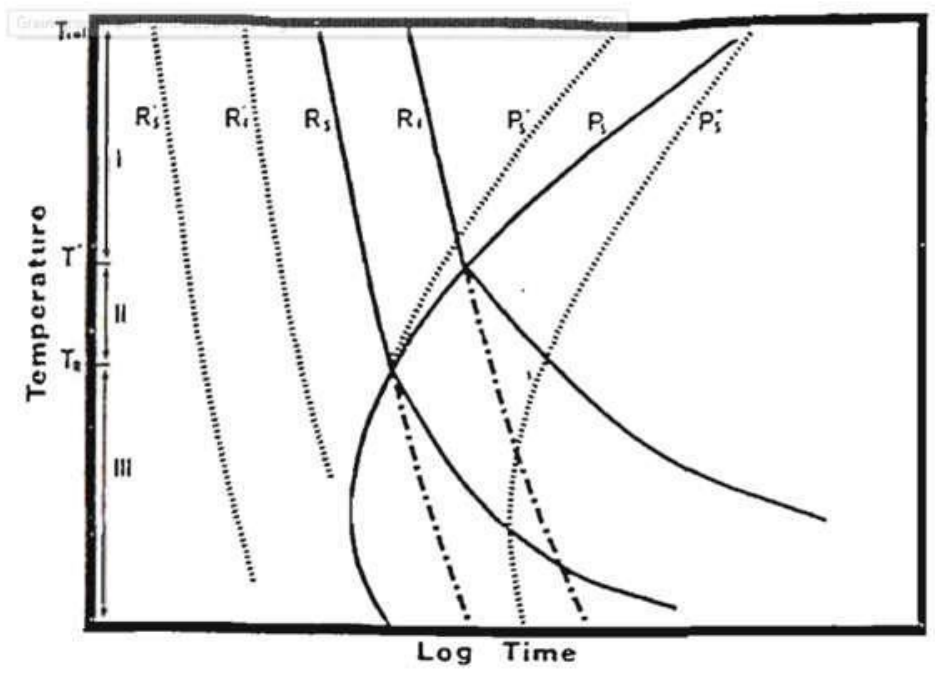

Figure 4: relation between recrystallization and precipitation of HSLA

Tsol $=$ Solution Temperature

$\mathrm{T}=$ Temperature below which precipitation/recrystallization complete

$\mathrm{TR}=$ temperature below which recrystallization occur before precipitation

Above figure shows three regimes:

First represents the initial austenitize process (Above Tsol) when recrystallization is done before precipitation (roughing deformations during TMP) when temperature between Tsol and $\mathrm{T}$.

Second represents delay time period in TMP. In this precipitation appear after partial recrystallization process.

Third represents the precipitation before recrystallization process occur (Finishing Deformation in TMP). Precipitation will cause pinning action (surrounds grains) and retarded recrystallization so that it may not occur after deformations. 


\subsubsection{Phase transformation of Micro-alloyed steel in TMP}

Decomposition of alloy upon cooling depends various factors like deformation amount before Tnr, alloy composition before transformation, precipitation amounts, cooling conditions etc. Transformation of Micro-alloyed is retarded due to presence of micro-alloying elements. Micro-alloying elements increases Ar3 (Austenite transformation temp.) which decreases carbon diffusion and retard transformation. Segregation of micro-alloy elements decreases the phase boundary and retard transformation. If Ar3 (Austenite transformation temp.) decreases, it refine grain size, increases dislocation density, decreases precipitation and retains solute in solution.

Austenite can be transformed into different phases i.e. bainitic, ferritic, martensitic or any combination, depending on steel composition. A schematic microstructure transformation types is given as following:

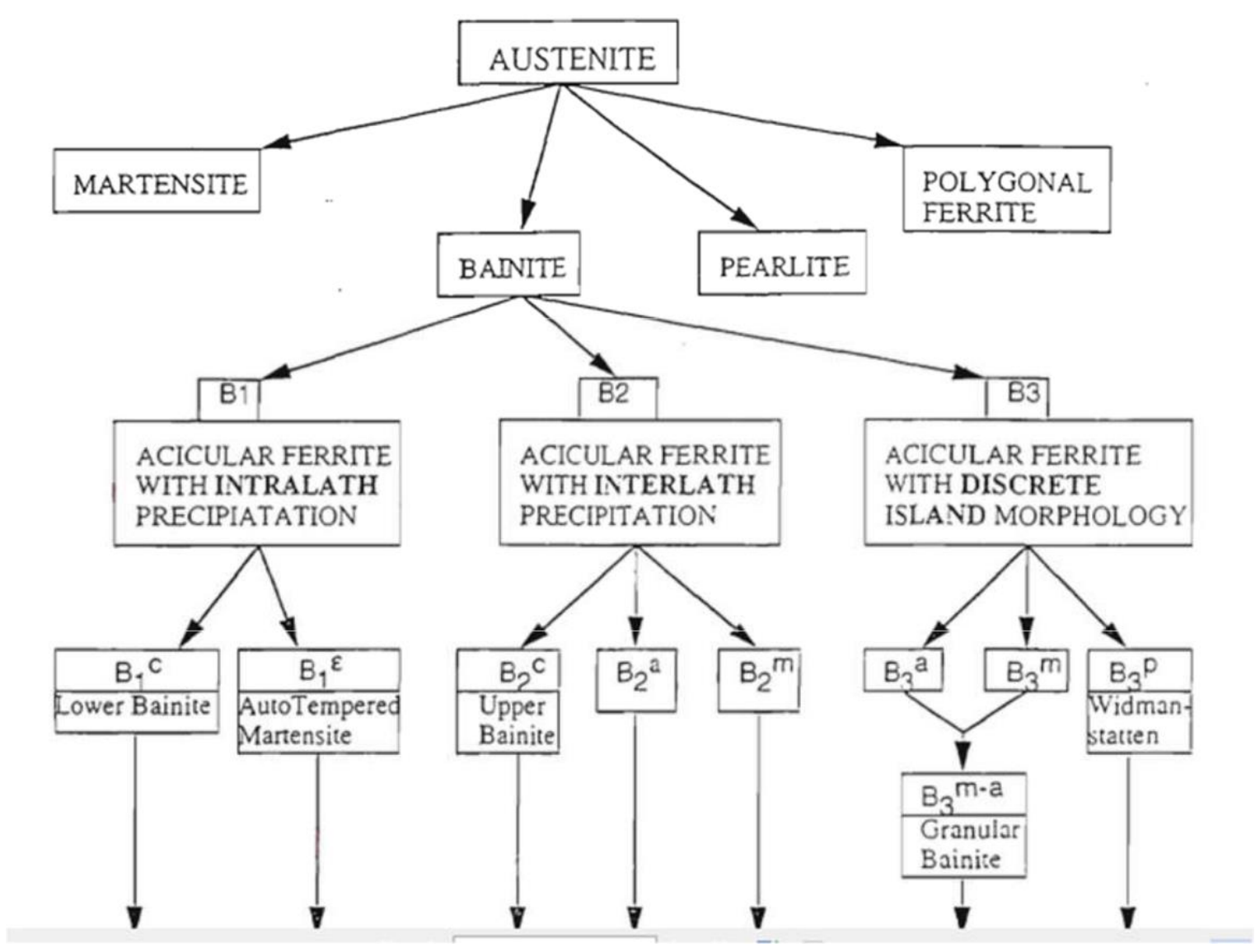



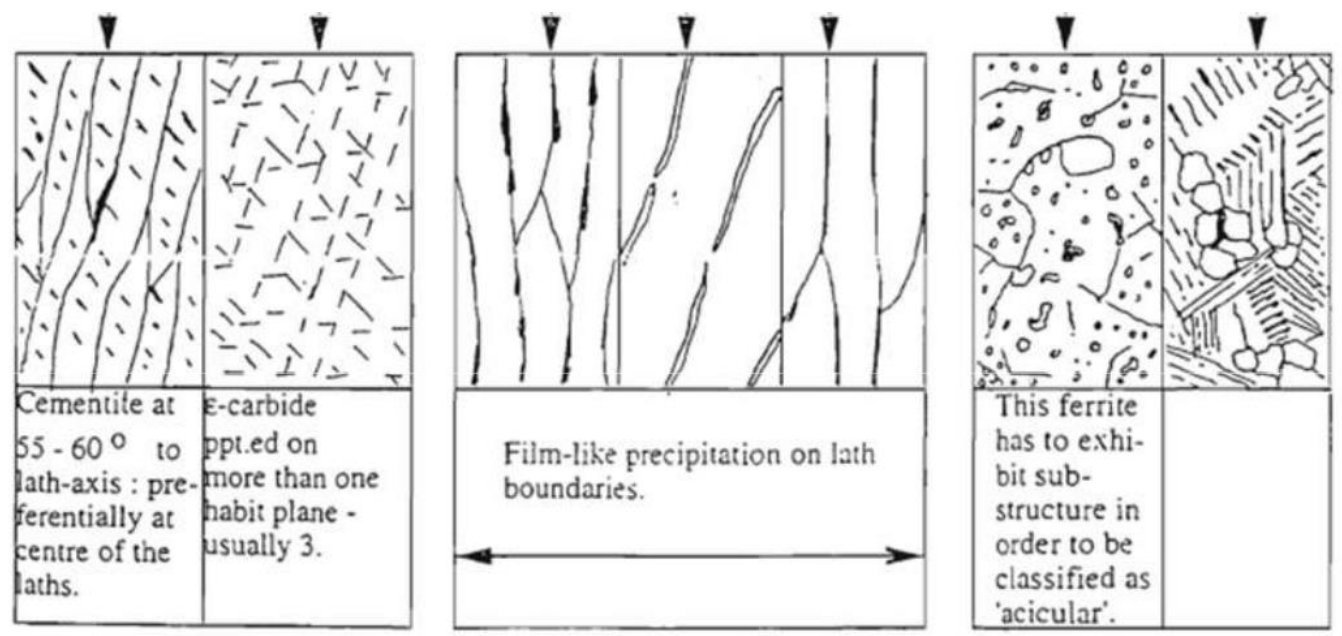

$p=$ pearlite,$m=$ martensite,$a=$ austenite and $c, \varepsilon=$ carbide

Figure 5: Micro structure Detail and Classification

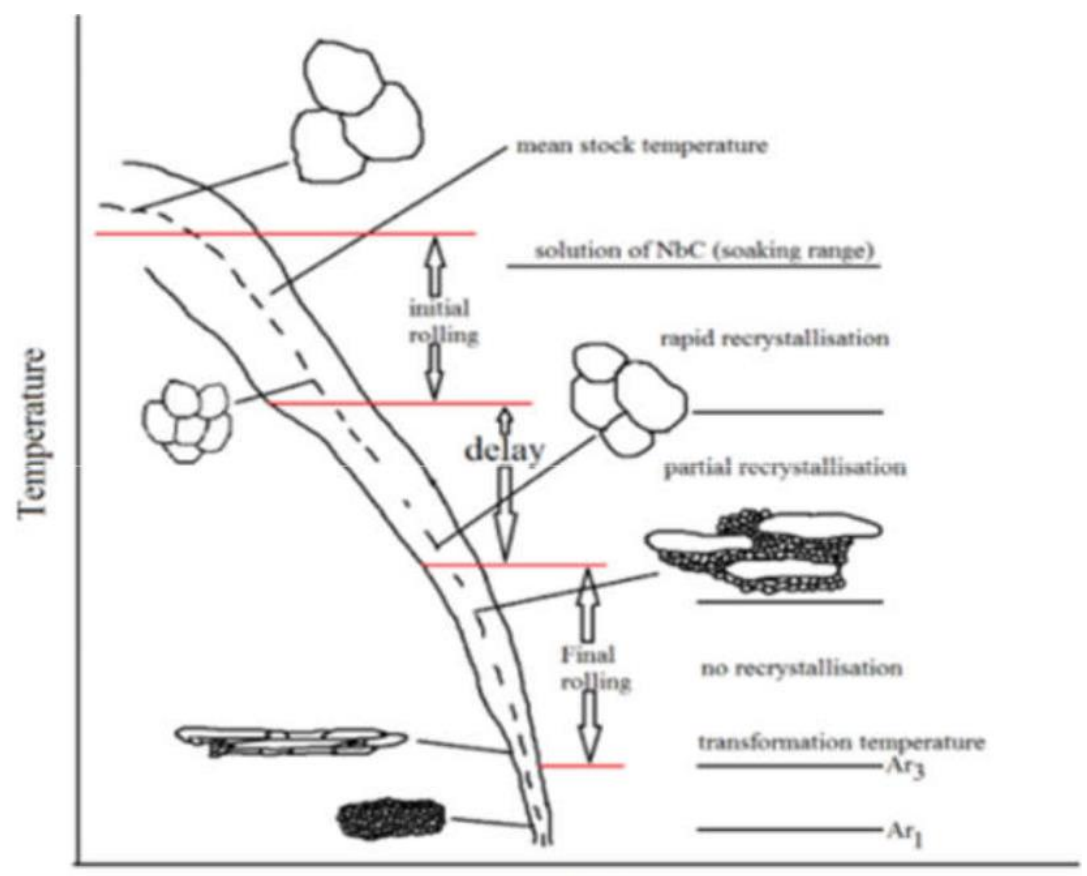

Time

Figure 6: Schematic diagram showing various processes during controlled rolling

\subsubsection{General HSLA Manufacturing}




$\rightarrow \quad \rightarrow \quad / \quad \rightarrow$

$\rightarrow h \quad h$ 


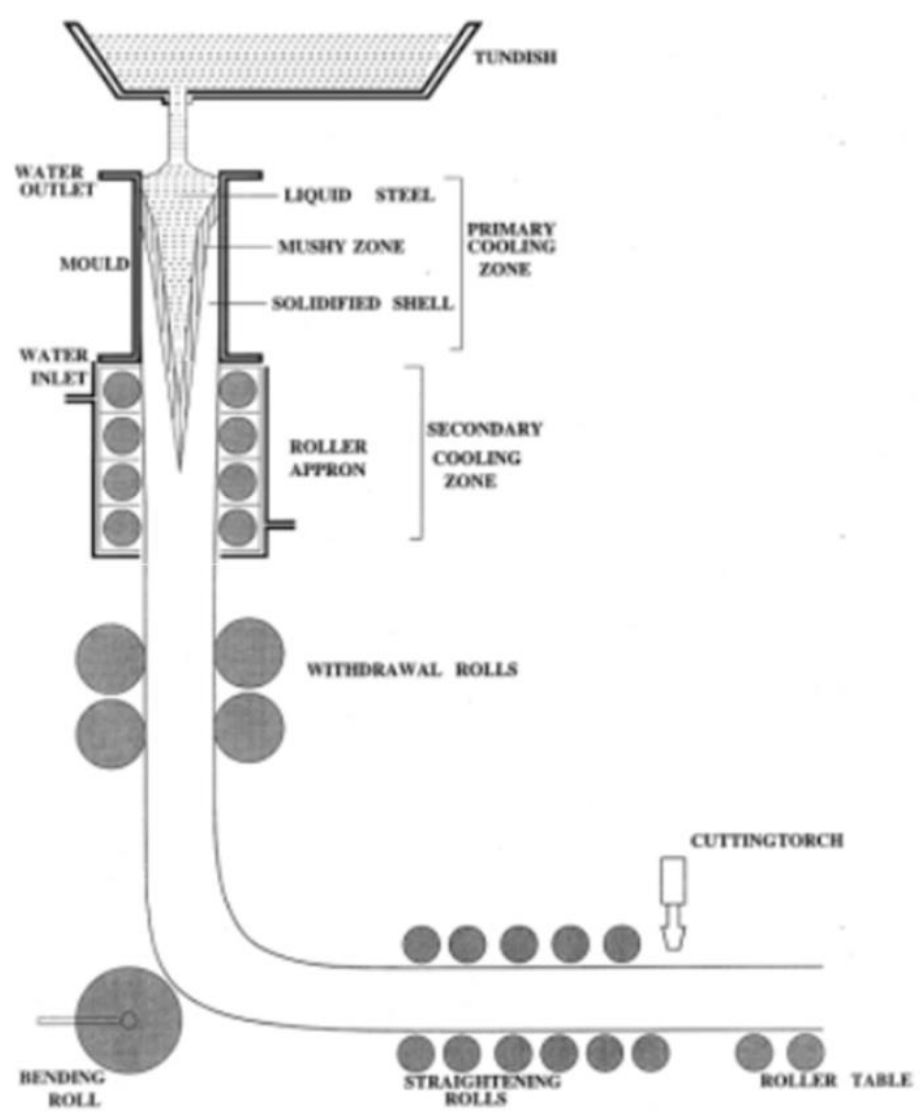

Figure 7: Continuous casting Process (HSLA)

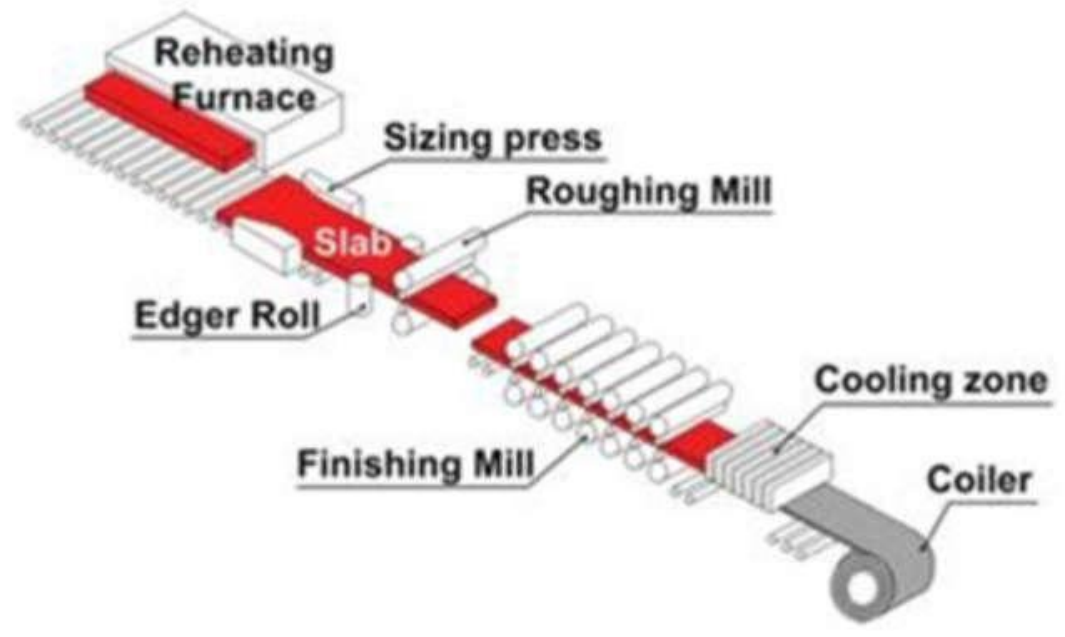

Figure 8: Hot Rolling process diagram

(Wang, 2017, Manohar, 1997, Mattes, 1990, Sallez, 2014, Bitterlin et al., 2016, KONSTRUKCIJSKA, 2011, Mandal, 2015, A Qaban, 2019) 


\subsection{Steel Manufacturing Process}

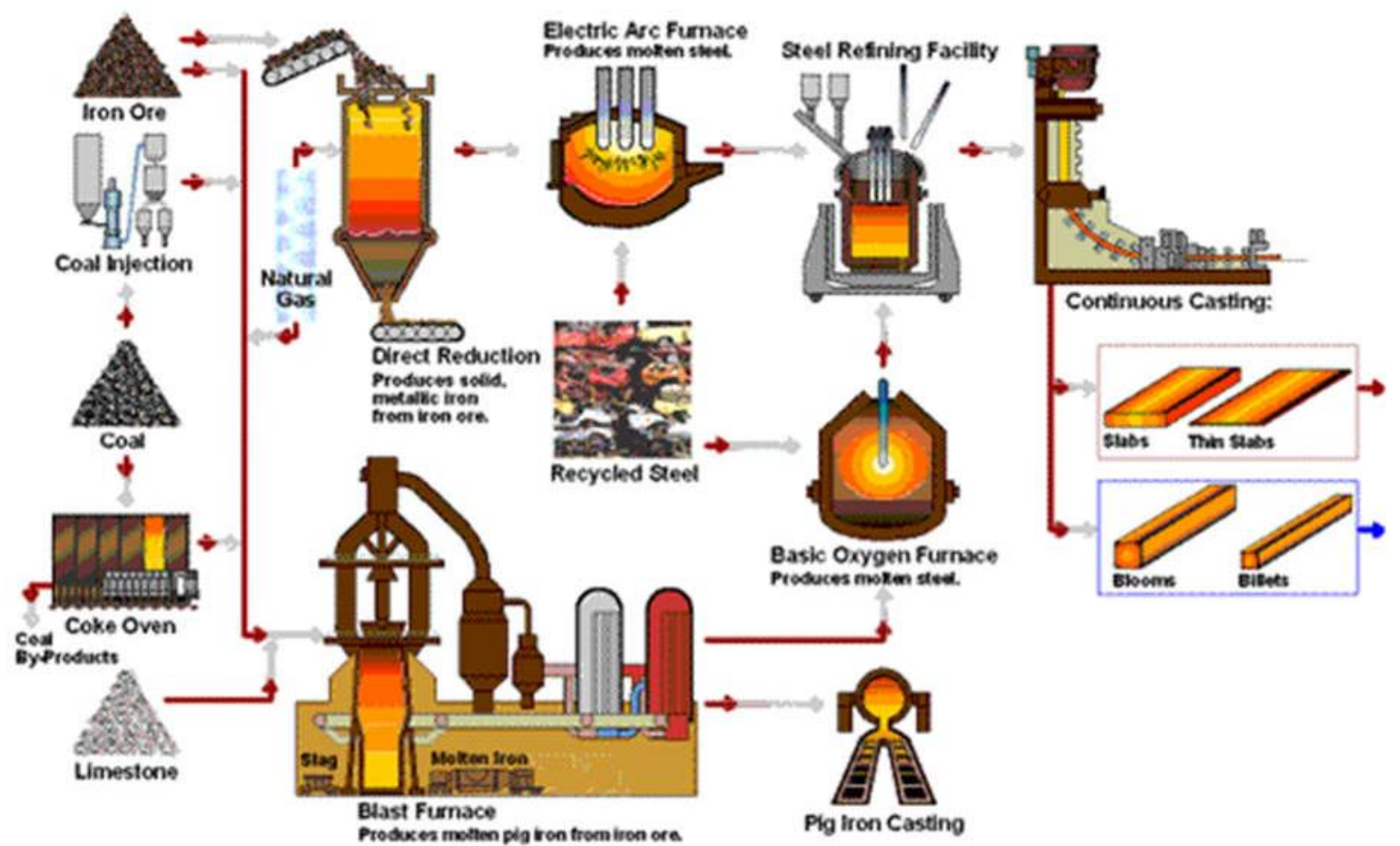

Figure.9a: Steel Manufacturing Process Diagram (Flowlines)

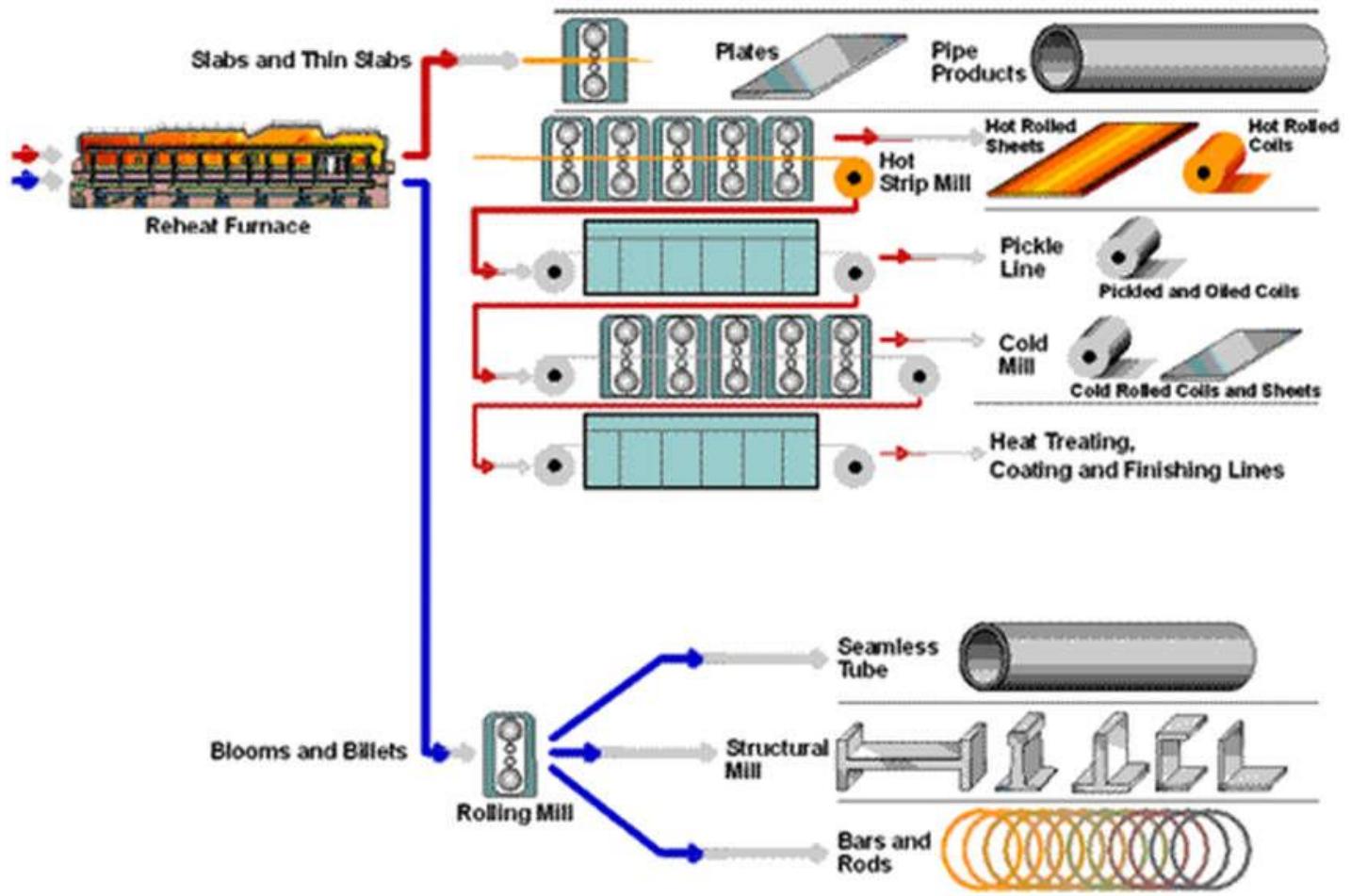

Figure 9b: Structural Shapes Formations 
(AISI, 2020)

Steel manufacturing is done through high temperature stages (Refer above figure) which can cause the abnormal grain size growth of the steel particles which can directly alter the Characteristics of the metal steel and can death the purpose of use for which it was manufactured. Therefore either in HSLA ingots or Steel manufacturing, high temperature can cause abnormal grain growth if exceed certain level of temperature. Therefore, it is logical to know process of grain growth, its abnormality, grain size and its control to get most of the benefit and use.

(Wang, 2017, Shirdel et al., 2014, Manohar, 1997, Bickford, 1998, Long and Wodarski, 2010)

\subsection{Extent of Problem (Case Study) | Understanding Case problem after overviewing the Literature}

High strength steel alloy is use for the extraction process. For HSLA pipes and equipment, HSLA-large size ingots are required. While dealing Large size ingots at high temperature either in manufacturing or rolling process, cracking phenomena is producing during open die forging process or after forging during heat treatment operations. The case study is to investigate the cause of cracking. The cracking is expected due to the Abnormal Grain Growth, Precipitation or segregation of the Metal-Alloy Particles.

Segregation in alloy can causes discontinuity in microstructure. Segregated and banded microstructure modify the grin causing brittleness of the alloy. Larger the ingot larger will be the segregation and larger will be the brittleness of the material to crack. Segregation is unable to remove during heating and forging.

Literature cleared that mechanical properties are developed as a result of secondary phases (Recrystallization/precipitation etc.). It might be possible, in secondary phases (or precipitations), there can be precipitations like $\mathrm{MnS}$ etc. which occur at or near the boundary to cause the cracking. As the expanded products precipitation is at boundary, therefore longitudinal or transverse cracks are easy to produce to compromise the toughness of the material. So, if we control the formation of any expanded precipitation, we can restrain this way of cracking which can be done with the help of silicon or calcium as they have more affinity for the sulfides than manganese.

Abnormal grain growth occurrence through any of the reason, will directly impact the toughness by contributing change in microstructure. Abnormal grains have smaller boundary 
length as compared with normal grains which effect the ductility of the metal alloy and remove the resistance of Metal (steel) to stress (deformations/strains) comes which can be an easy path for the cracking either during forging or heat treatment processes. Upon compression of $\mathrm{NiCrMoV}$ medium carbon steels, dynamic recrystallization may occur which refines the grain size and carbonitrides formed under dynamic precipitation stops abnormal grain growth. In this scenario, cracking will not be feasible during forging so this cannot be the expected failure.

The subjected ingot failure alloy have nickel and chromium upto $2 \%$ by weight in proportion. AISI 4340 steel crack during forging and melt during over heating at grain boundaries. He et al shows the similar behavior for the Ni-Cr-Mo-V steel in which cracking caused due to the high strain and deformation rates during forging.

One other possible way of cracking may be due to the corrosion at interface of oxide and steel (Nickel added Steel) which may occur during the forging process. But this type of failure seems to be illogical to predict before a comprehensive study done about the cracking in a single type alloy metal.

All above was just for the better understanding of the case problem and predicting expected reasons and causes of cracking in large size ingots on literature basis. Ultimate causes can be clear only with experimental method in which different characterization techniques can be applied and microstructure of the subjected large size ingots is observed from deep analyze the change and concluding cause which causing cracking in ingots. Following section will be experimental based.

(Bitterlin et al., 2016)

\section{Experimental Section}

\subsection{Characterization Techniques}

Characterization techniques are used to review the microstructure and compositions of the sample tested in order to evaluate the cause of the failure. Following are some common techniques used for the characterization and testing of the material sample:

\subsubsection{Classical Techniques}

Optical and Scanning Electron Microscope

Electron Microprobe 


\subsubsection{Advance Characterization methods for nanoprecipitation}

Atom Prob Tomography

Small Angle Scattering (SAXS, SANS)

Optical Metallography

\subsubsection{Advance Techniques for Structural Failure}

Energy Dispersion Spectroscopy (EDS)

$\square$ XRay Diffraction (XRD)

$\square$ Automatic Crystallographic Orientation Mapping (ACOM)

$\square$ Transmission Electron Microscopy

In this case study, X-ray diffraction and Electron Dispersion Spectroscopy is performed to analyze the microstructure and composition of sample.

(Bitterlin et al., 2016, Sallez, 2014)

\subsection{Experiment}

\subsubsection{Sample Collection}

Sorel Forge Inc., Quebec, Canada collected and forwarded sample for the testing procedure.

\subsubsection{Composition}

Composition of the sample ingot is given as following:

Table 2: composition of sample taken

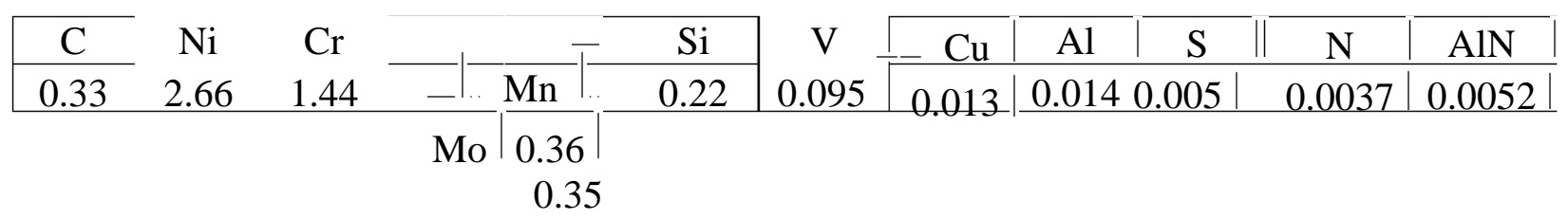

\subsubsection{Cracked Sample Preparation}

A 56" diameter and 20 tons ingot casting is prepared for the testing. The sample is subjected to the 7 passes through open die forging process and then heating treatment were applied comprising quenching, tempering and normalization of the sample. Heat treatment were carried out at $900^{\circ} \mathrm{C}$. The resultant large size forged ingot under high temperature treatment showed cracks 
as per expectations. Standard Procedures and conditions of forged and heat treatment procedures were applied with no error in record.

\subsubsection{Procedure}

A cracked sample is prepared as discussed in above paragraph.

$\square$ Surface oxides removed by machining process and then sample forwarded for ultrasonic and dye penetrant nondestructive analysis. Result of this test is discussed in figure 1.

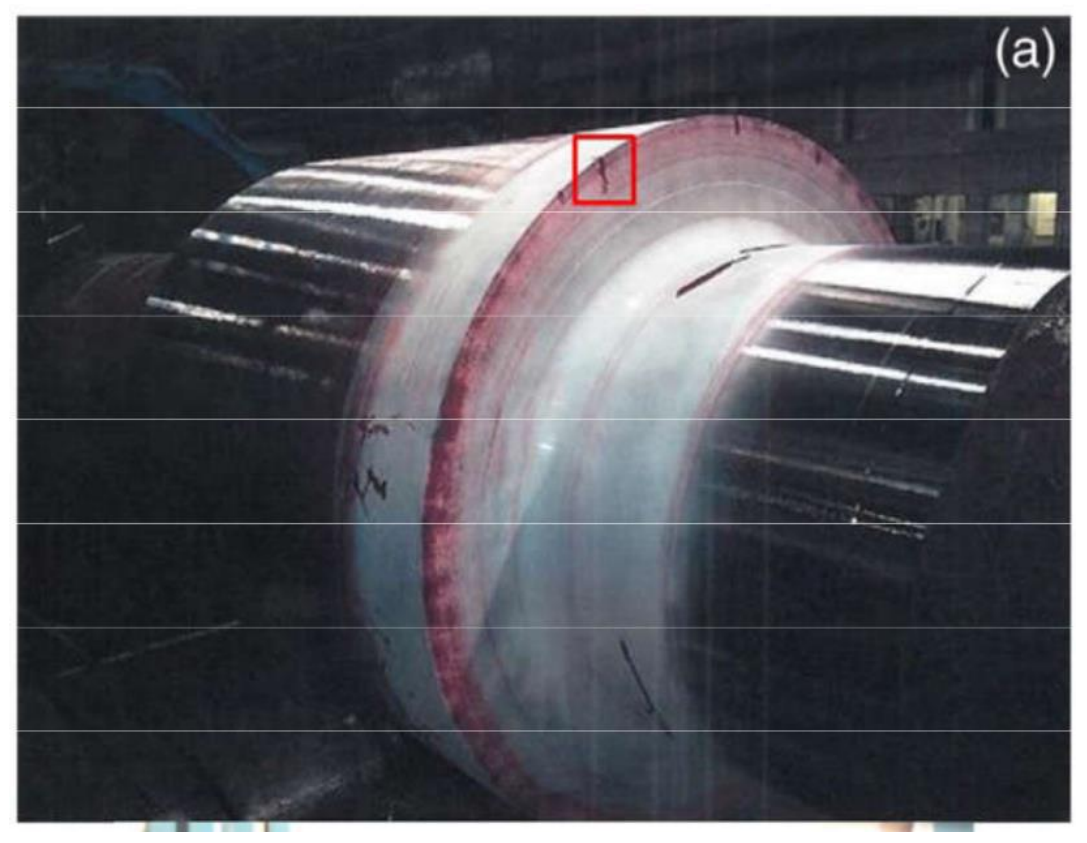

Figure 10 (a): a result after dye penetrant testing 


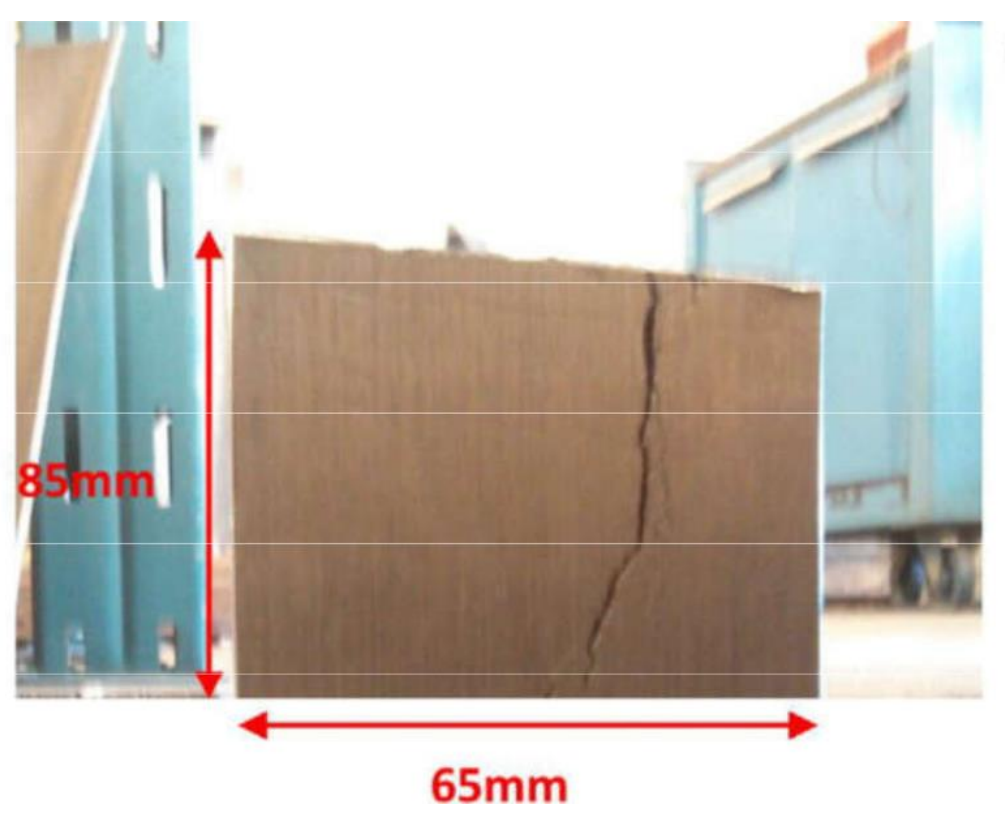

(b)

Figure 10 (b): Dimension of machined out block

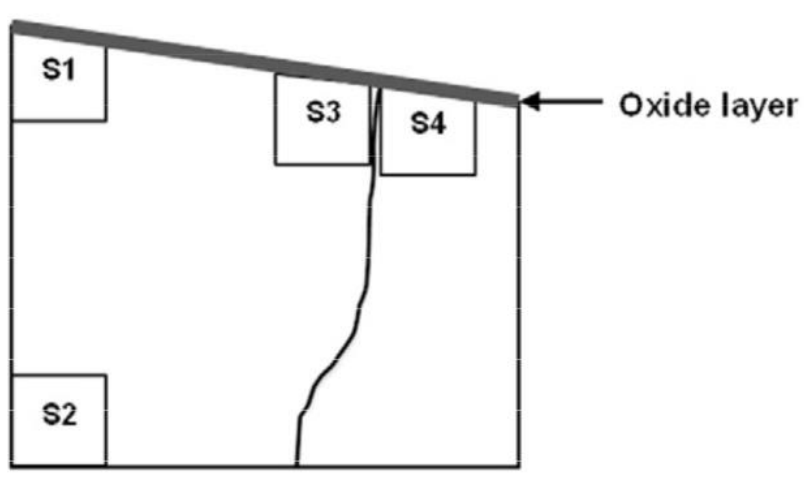

(C)

Figure 10 (C: 4-small specimens taken

Figure 10a shows the large number of cracks of the ingots of which cause has to be investigated in the other testing results. Figure 10a also defined the position of forged ingot where a large block machined out of the sample. Whereas Figure 10b is showing the sample dimensions as $8585 \times 65$. Figure $10 \mathrm{c}$ shows the 4 -specimens taken from machined out block at crack locations for further testing procedures. These samples were taken at cracked locations, in its vicinity and far off cracked zone and near the surface.

4-Samples polished with Silicon Carbide paper for 200 to 1200 grade then resulted with 1 micro meter paste. This rubbing is done to clear the austenite grain boundary which is the most challenging part in test sample preparation. 
Various solutions were proposed in literature without any conclusion. Picral solution was developed for the current investigation.

$\square$ Picral Solution: 10 gram of picric acid with few drops of zephiran chloride was heated at $83^{\circ} \mathrm{C}$ to prepare a solution which will act as wetting agent.

4-samples taken in previous steps, were immersed in Preparation of Picral solution for 105 seconds.

Sample removed from solution and then cleaned with water and then cleared with the methanol to remove picric acid residue.

After drying of the sample, cracked surface was made cleared and reviewed Beaujard reagent at room temperature for 240 seconds.

Beaujard reagent consist picric acid with few drops of sodium alkyl sulfonate, the solution will be acting as the wetting agent.;

Literature clear that Beaujard reagent reveal grain boundaries for the observation of the microstructure.

The sample was observed for microstructure over Confocal Microscope Olympus LEXT 4100.

Standard ASTM E12-112 procedure was used for the determination of grain size whereas image analyzer MIP 4.2 were used for obtaining the grain distribution.

Scanning Electron Microscopy was done on SEM Hitachi TM3030 at $15 \mathrm{kV}$ equipped with Energy Dispersion Spectroscopy. This have equipment have ability to investigate for detailed microstructure, perform chemical analysis, oxide layers and inclusions.

X-ray diffraction were performed over X'Pert ${ }^{3}$ MRD PANalytical with Cu-K energy radiations with step size (2-theta) of 0.033 .

To investigate the presence of phases between 500 and $1600^{\circ} \mathrm{C}$ of investigated steel, Thermo-Calc-thermodynamic software was used. The results were also compared with the experimental results. 
4. Results:

\subsection{Secondary Cracks formations}

M. Bitterlin et al. / Engineering Failure Analysis 68 (2016) 122-131

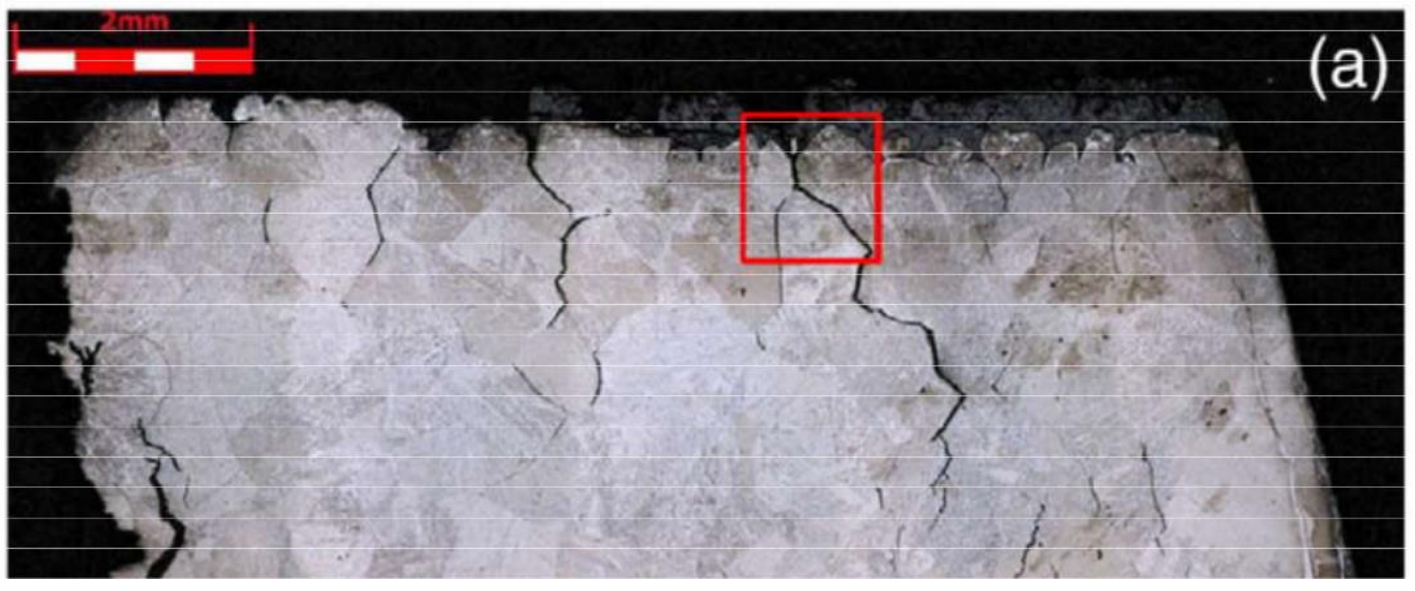

Figure 11 (a): S4 Sample Surface (Micrograph) 


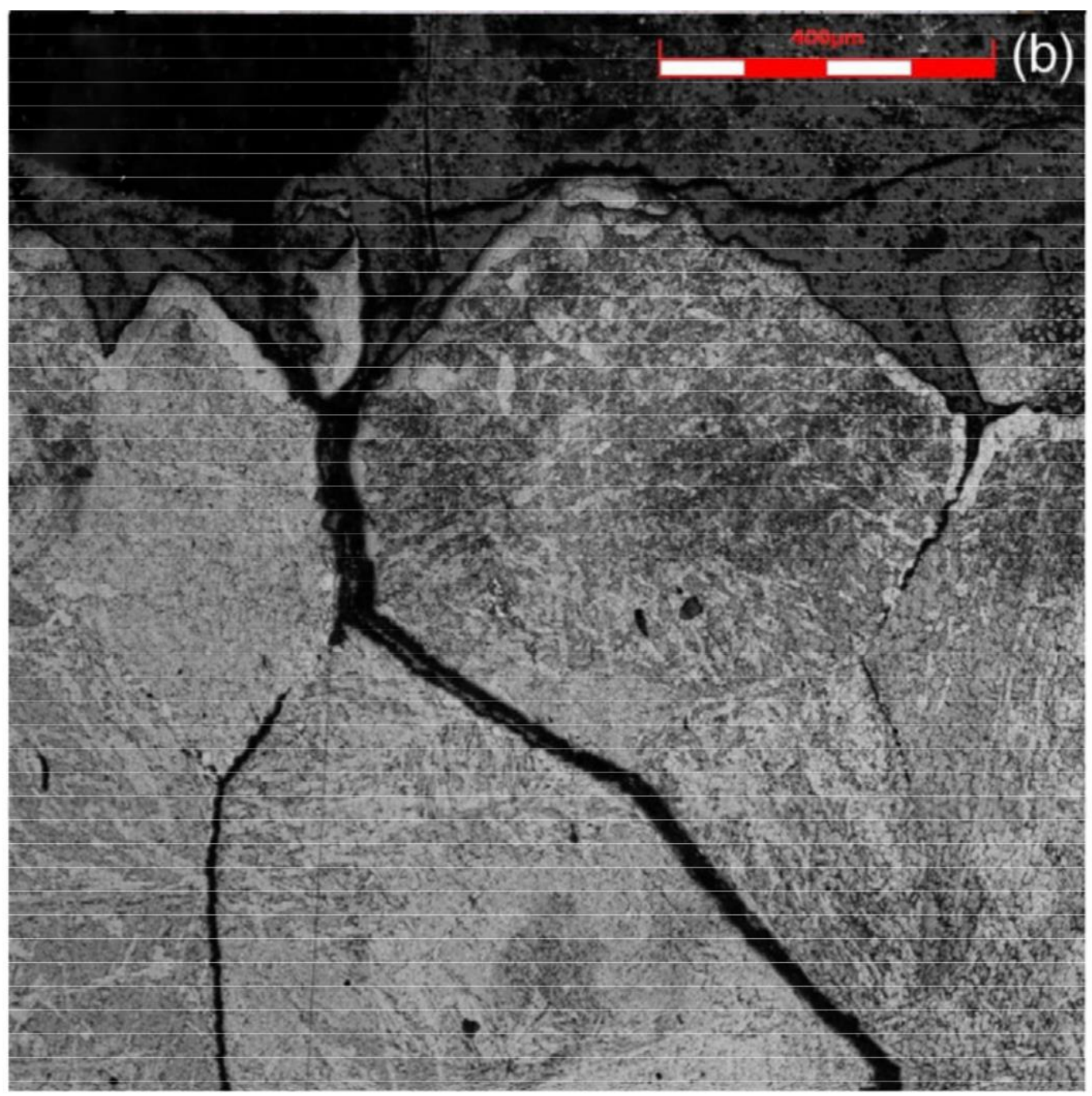

Figure 11 (b): Oxide Following Grain Boundaries

Secondary cracks were observed with the large cracks as shown in the above figure (a) taken by the optical microscope. From above figure (a) it is clear that large cracks followed grain boundaries whereas secondary one were inter and trans granular. In S4-Sample (Figure a) Large cracks were developed from surface and then join the grain boundaries.

\subsection{Orientation of Bainite}

Above figure (10.b) reveals the cracks on extreme left side of the sample. Near surface cracks sample were examined in which orientation of bainite laths and severe contrast changes was clearly depicted. This kind of cracks originating from one region to other is related to grain sizes. 


\subsection{Oxides formation at boundary}

In above figure (b) from micrograph, oxides are visible at grain boundaries which in return creating voids and grains separation.

\subsection{Grain Size Measurements}

Table 3: Grain Size found in Different Zones

\begin{tabular}{|c|c|c|c|}
\hline Zones & Depth from Surface & ASTM Grain Size & Average Grain Dia ( )) \\
\hline S1 & 0 & 1 & 250 \\
\hline S2 & 85 & 6.5 & 35 \\
\hline S3 & 0 & -1 & 550 \\
\hline S4 & 0 & -0.5 & 425 \\
\hline
\end{tabular}

Zone adjacent to crack had average grain size as $500 \mu \mathrm{m}$. Above table shows grain size for all zones which shows the large size of grains at surface of the samples.

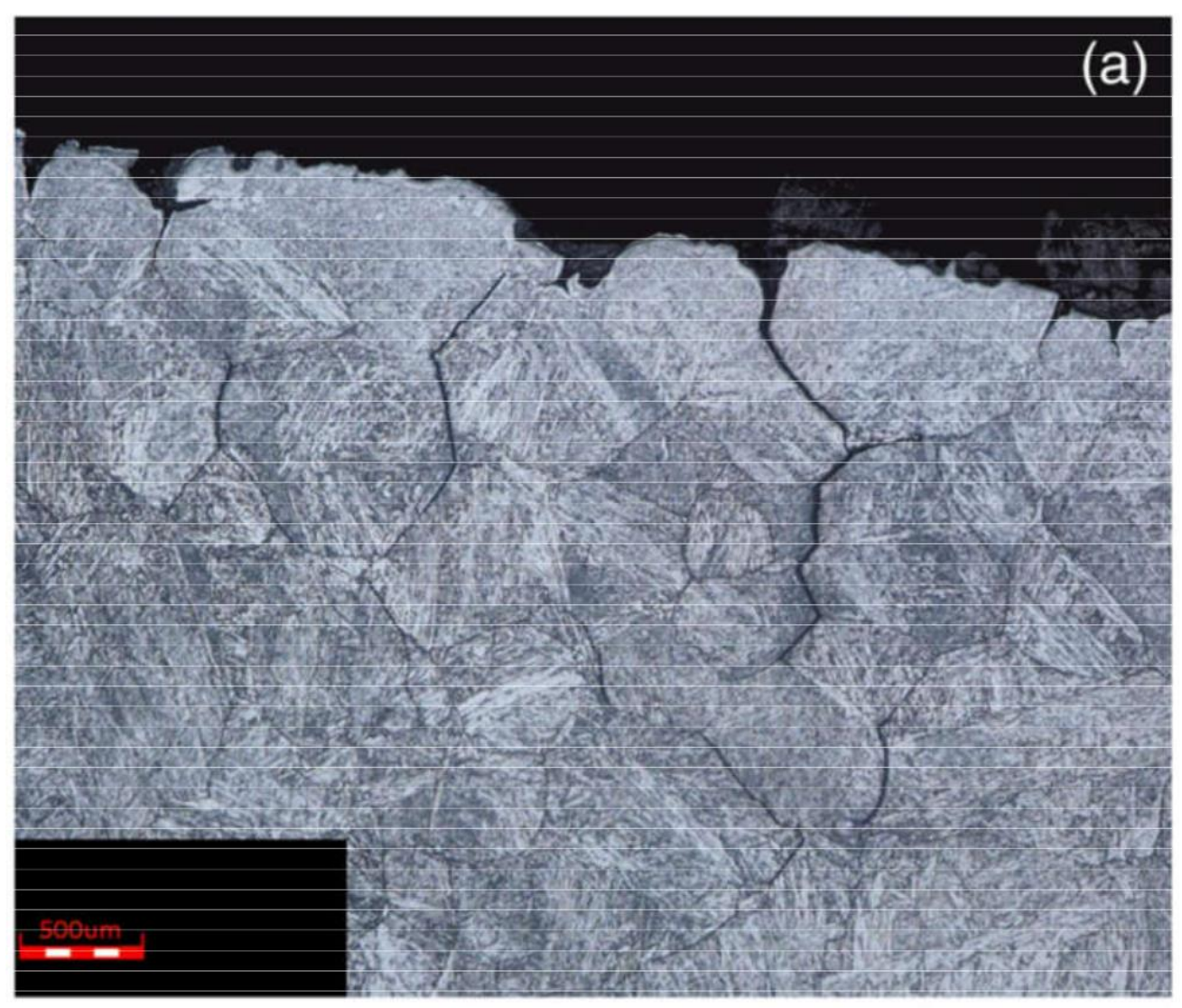




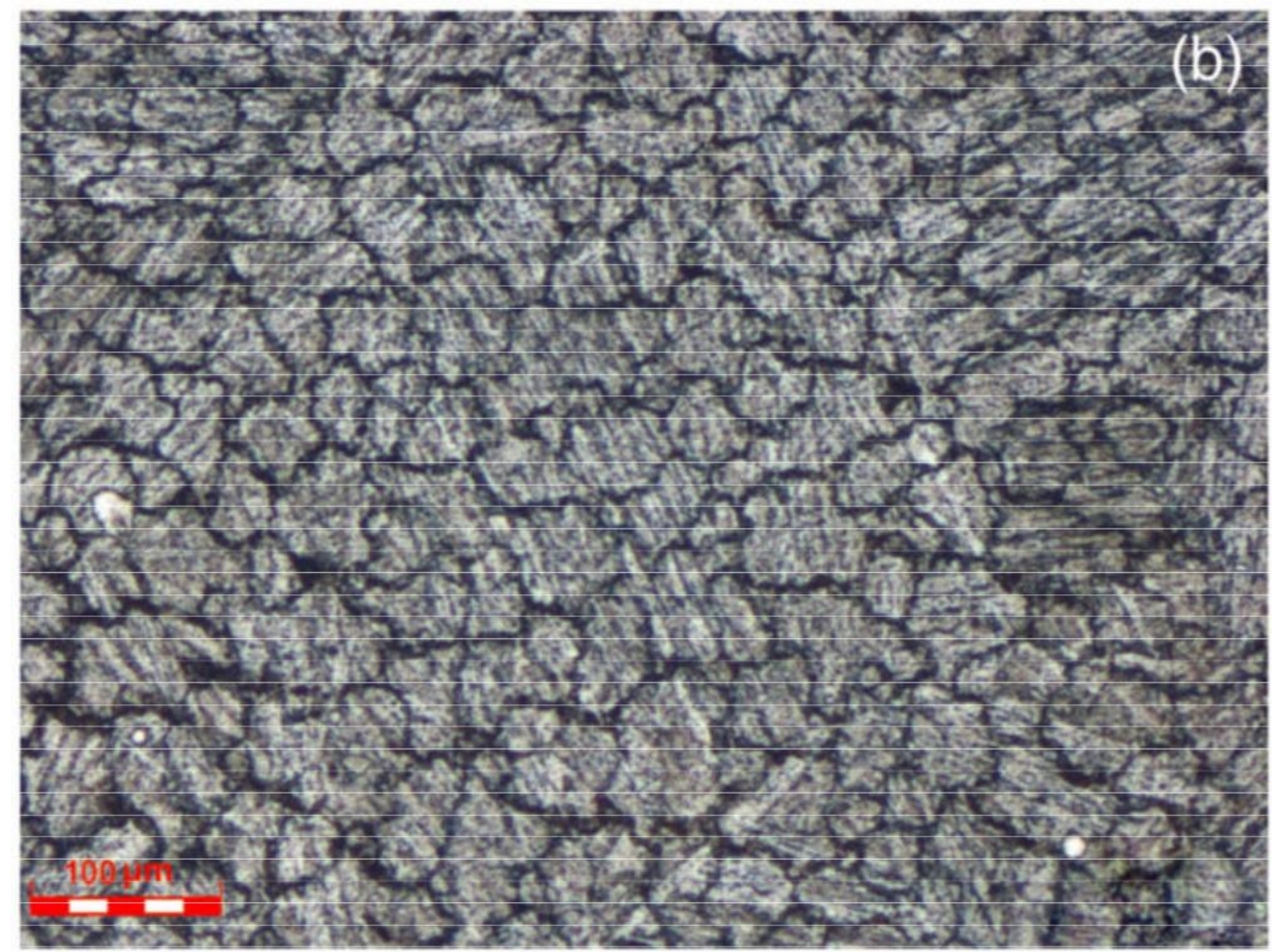

\section{Figure 12: (a) microstructure cracks on S4-surface (b) Grain Size in S2}

Above figure shows bainitic microstructure differentiated by plates and laths. Intergranular cracks can be seen easily in pictures. It can be clearly seen that, grain size near crack is larger than the others which is clearly the indication of abnormal grain growth occurred near cracking zone, obviously the reason behind failure. Above table and figure depicts the 10 times smaller grains away from the surface as compared with surface grains.

\subsection{Secondary Phases formation}

Thermo-calc carried out to simulate the phases between 500 and $1600^{\circ} \mathrm{C}$. Liquidus equilibrium transformation lines were determined using above figures. After examining above figure (b), it was noted that the precipitates consist of carbides and chromium and vanadium alloys. MnS and AlN were present in lower amounts as 0.029\%. All this viewed under ThermoCalc Analysis. 


\subsection{X-Ray diffraction}

First oxide layer was examined under X-ray diffraction and it cleared the presence of magnetite and Nickel (Iron)

M. Bitterlin et al. / Engineering Failure Analysis 68 (2016) 122-131

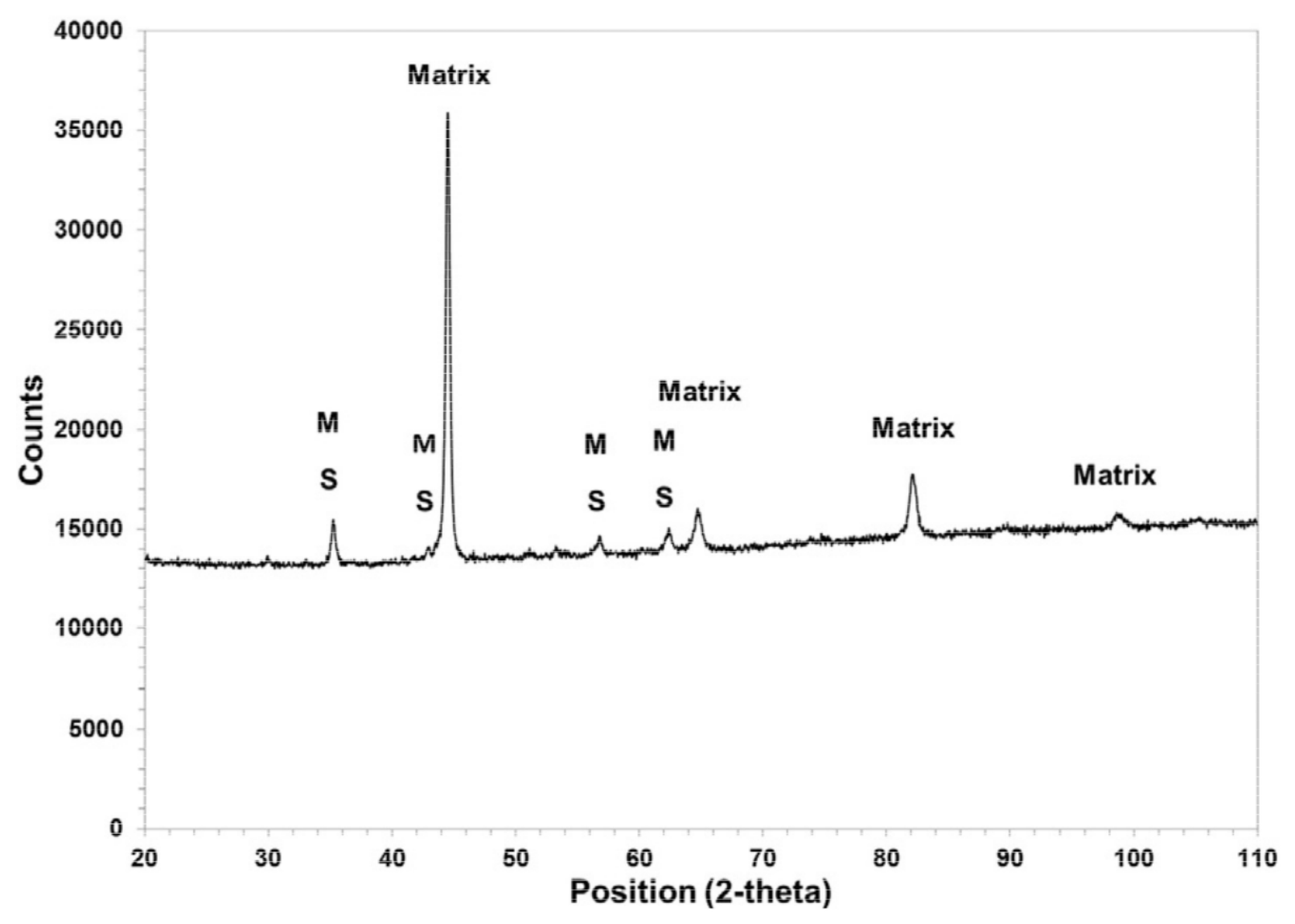

Figure 13: X-ray diffraction of oxide layer 


\subsection{Energy Dispersion Spectroscopy}

Second phases were analyzed under EDS technique and it was found the presence of nickel oxide and iron oxide.

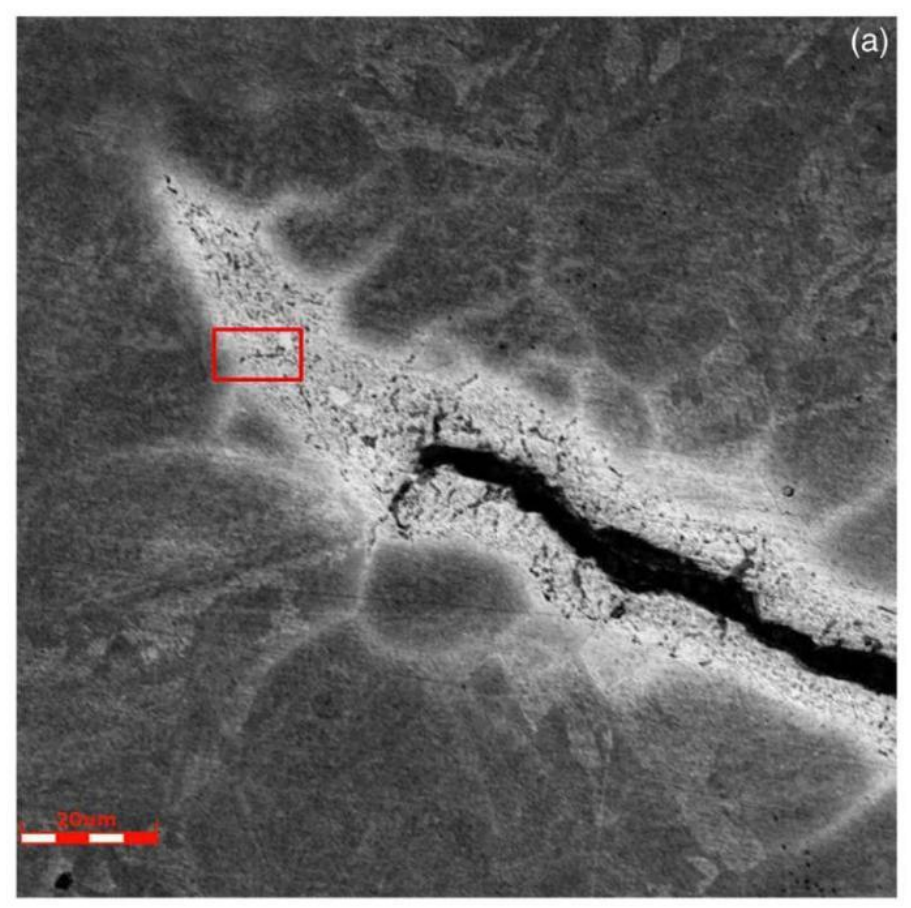



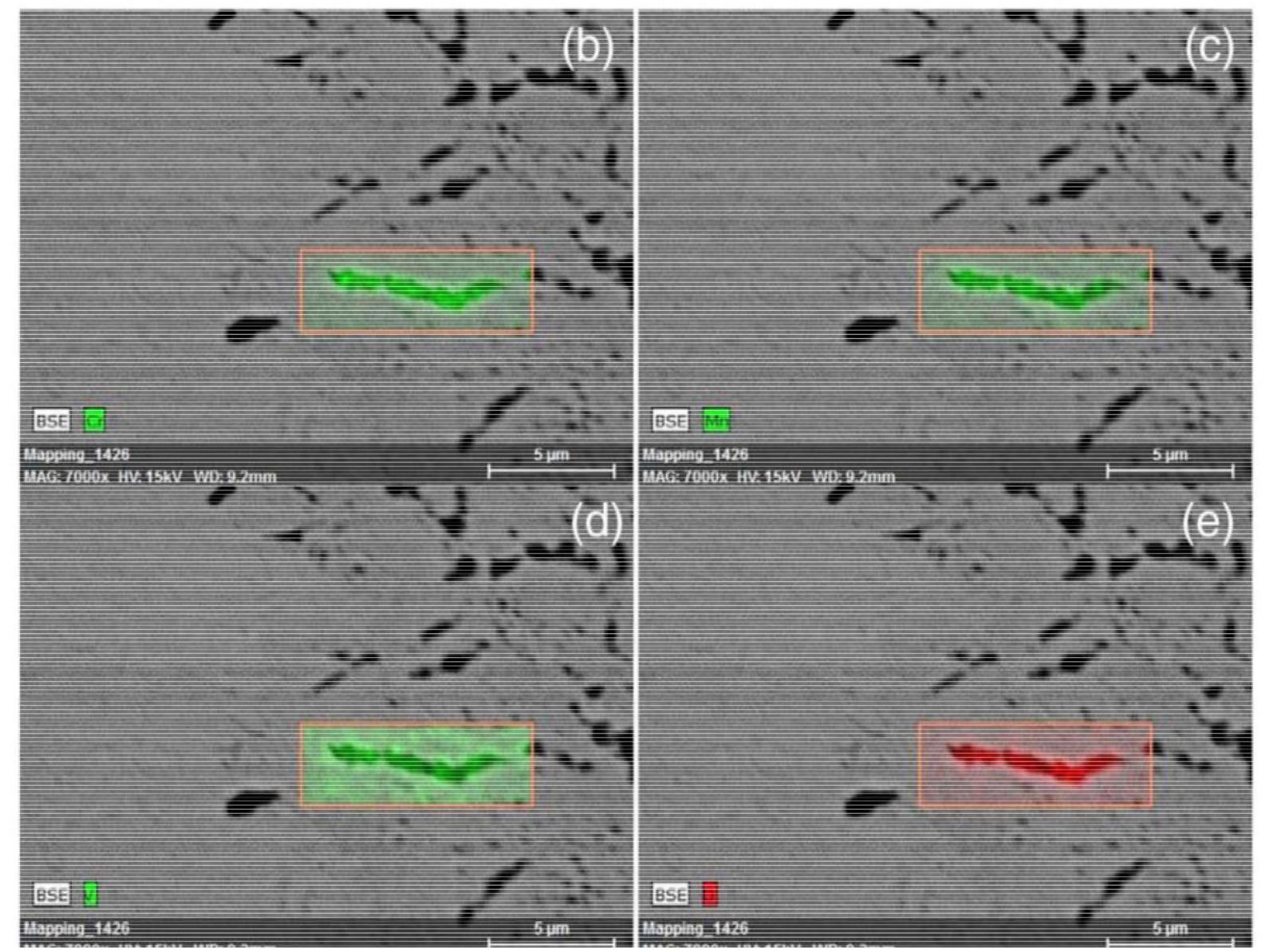

Figure 14: Microstructure of crack was revealed using the Beaujard reagent.

(a)showing Base crack, rest parts having $\mathrm{Cr}, \mathrm{Mn}, \mathrm{V}$ and $\mathrm{O}$

\subsection{Scanning Electron Microscopy}

Following figure explaining the base crack. Etching on the grain boundaries occur due the Beaujard's reagent reaction with oxide scale depleted Carbon-zone. EDS analysis investigate the preence of chromium and manganese oxide in precipitates. 


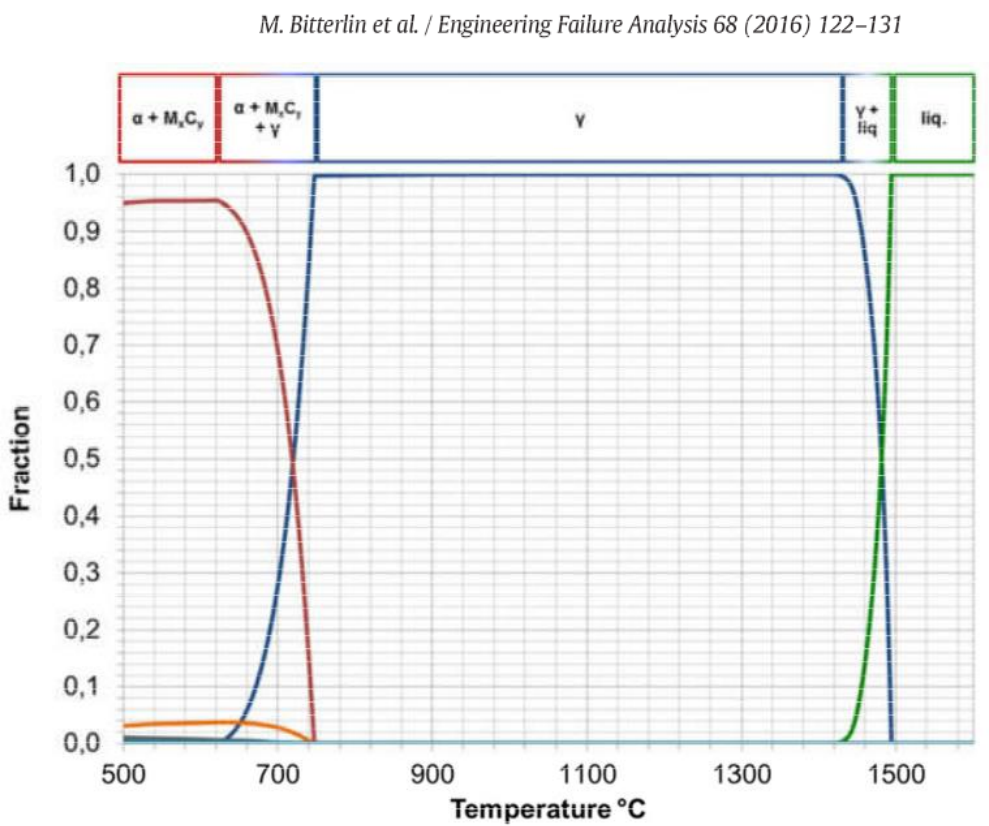

(a)

$$
\begin{aligned}
& \text {-Austenite } \\
& \text { - Ferrite } \\
& \text { - Liquid } \\
& \text { - AIN } \\
& \text {-MnS } \\
& \text {-M23C6 } \\
& \text {-M7C3 } \\
& \text {-MC }
\end{aligned}
$$

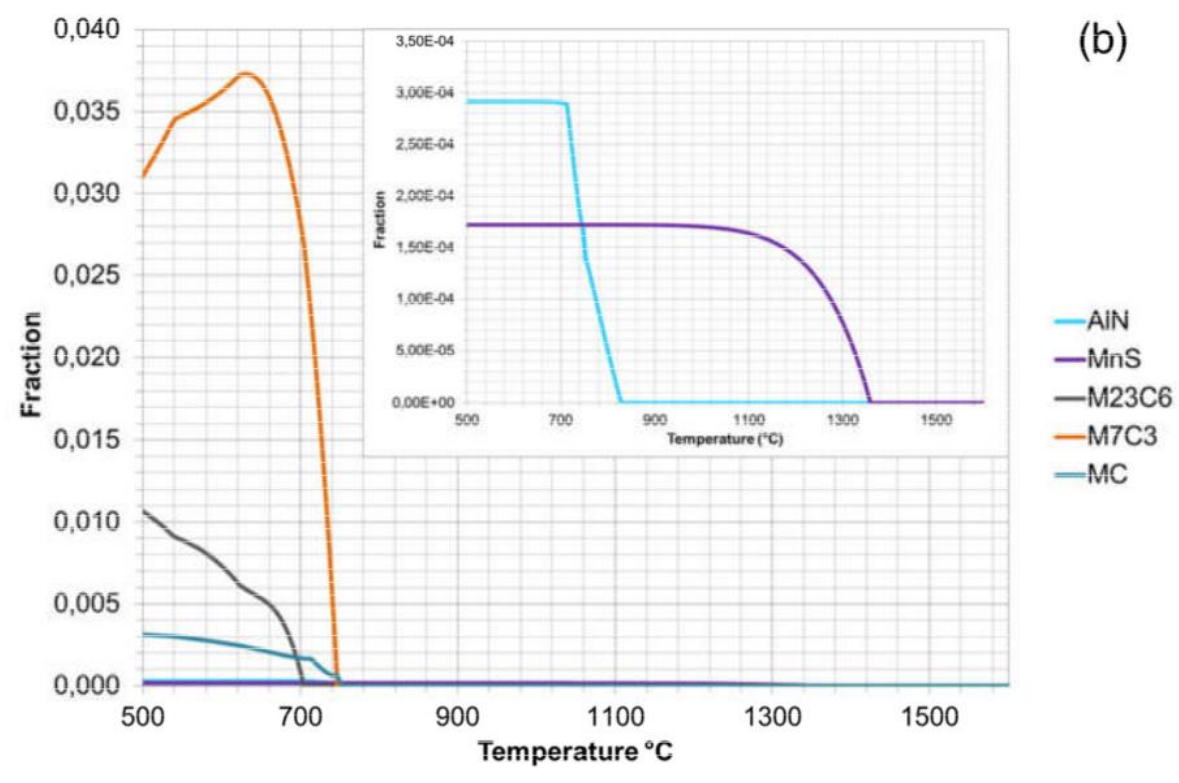

Figure 15: (a) whole simulation (b) Close up precipitate on precipitate proportions.

\subsection{Line Scan EDS Analysis}

Following figure clearly depicts the high amount of chromium present in oxide layer on sides of the crack, therefore chromium is also the cause of failure. 
M. Bitterlin et al. / Engineering Failure Analysis 68 (2016) 122-131

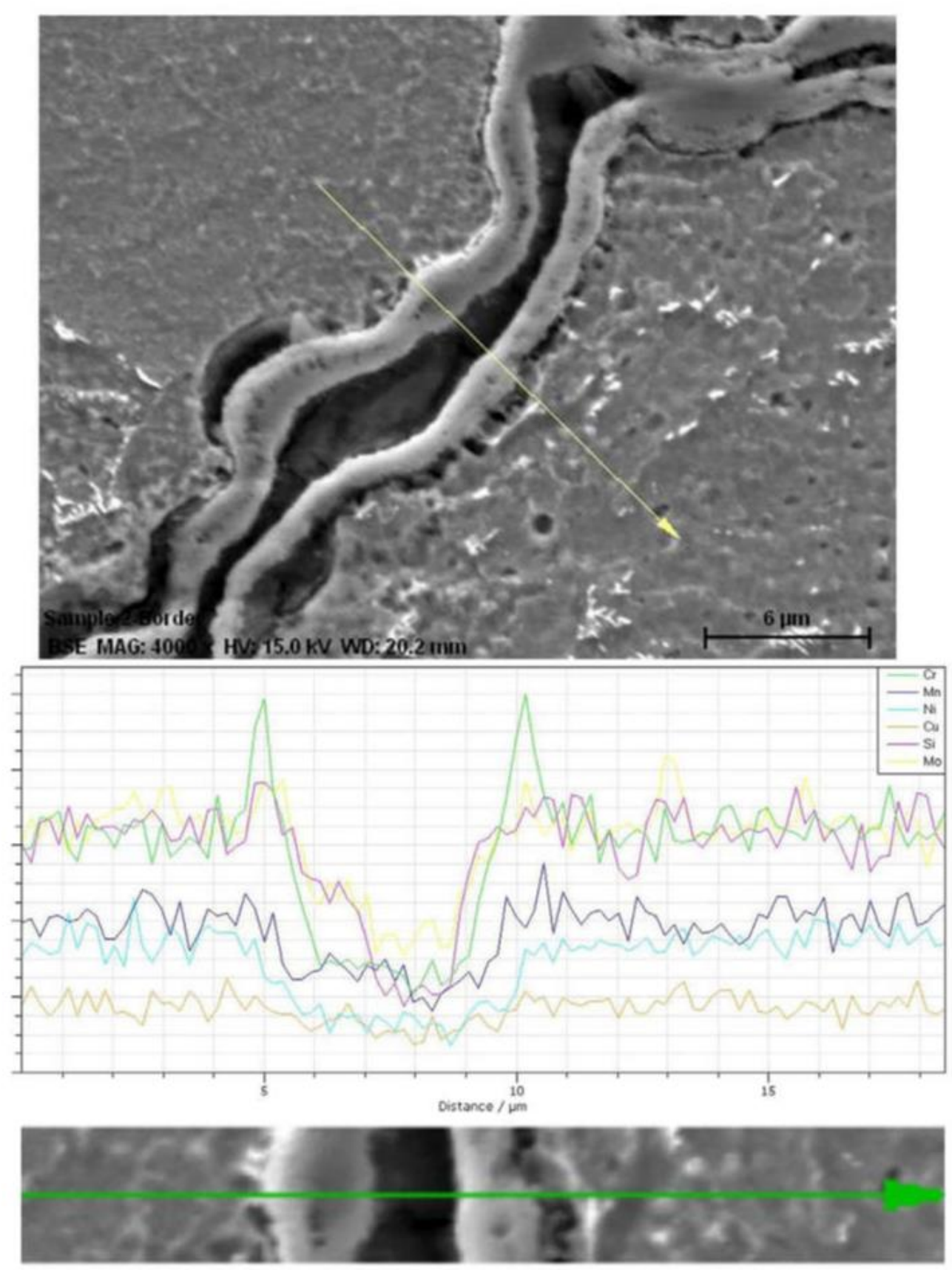

Figure 16: EDS analysis to know chromium amount

\section{Discussion | Possible Solutions}

In figure 11 and 12, large grains are present at surface and near the main cracks. It is

related to the 7-passes of forging. Grain growth is affected by the precipitation of carbides and nitrides. In 
tested steel most common precipitates found were Chromium and Molybdenum carbides. It is due to their low enthalpy of nucleation and growth. In thermo-calc simulations as referred in figure 13, there was the plenty of chromium precipitates instead carbides. even the small grains of carbides can be removed during etching process. Thermo-calc results in experiment were same literature study over carbides in microalloyed steel. Thermo-calc process also shows the formation of $\mathrm{MnS}(0.017 \%)$ or $\mathrm{AlN}(0.029 \%)$ under considered conditions.

Size of precipitates can be controlled in microstructure by mixing subjected steel with Calcium. MnS was present in precipitates which link with the sulfides preset in the solution. AlN was not traced during sulfides. High nickel content oxides present in the oxide layer. Nickel content in silicon containing steel is smaller than the base plate. Current experimental (EDS) study have not shown and deficiency in level of the nickel in surface zone. Chromium and manganese are present on the edges of the cracks as shown in figure 15 which means that there is no hurdle to delay grain growth etc.,

Oxides are formed due to re-heating process or cooling cycles during open die forging process. Manganese and Chromium elements are mobilized under high temperature during the diffusion process. Latter has 100 times greater diffusion than normal iron at higher temperatures. Manganese follow the same way, as it have more diffusion rate as compared to the iron or chromium element. Multiple oxides are formed at the boundaries near cracks. Over this cracked area, etching processed with Beaujard's reagent which shows the development of $10 \mu \mathrm{m}$ oxide layer and a zone $1 \mu \mathrm{m}$ formation of grains boundaries near the crack. Grain size at the base of crack is $15-20 \mu \mathrm{m}$ (small). Hence growth proceed with intrusion of the oxides from far surfaces. This result in the abnormal grain growth and cracking of the material. As shown in figure 11 and 12.

Etched Specimens were experimentally tested and it was clear through metallographic technique that there are oxides are present which grow through grin boundaries and intensities (depending upon Crack size and distance from the surface). Oxides formation is one of the root cause of cracking and make material fragile. Near surface zone, slab is characterized by a reduction in quantity of the alloying elements. In current sample no such phenomena seen where chromium oxides occur at cracks or near cracks. This is due to the fact that Chromium oxide has higher growth rate as compared to diffusion rate. EDS cross line Scanning Analysis shows in figure 16 
that chromium oxides have higher content at the boundary as compared with vicinity where, there is no change.

Deformation of the material occur at higher temperature during cogging process. Dynamic Recrystallization phenomena may occur when strain deformations applied. If the subsequent line phenomena not occur, rapid growth of grain may occur in result. These large grains may causes the abnormal grain growth ultimately affecting toughness and causing the cracking in material. Reheating process after deformation may cause the grain growth of ingot during forging which may cause the cracking. For recrystallization, sufficient deformations may require. Under static deformations, recrystallization will be dynamic for example interpass time between each re-heating stages.

Grain growth is the function of strains and temperature during static and dynamic recrystallization. Fast but small deformations can affect nearby surface zone of the material. Similarly in large ingots, during re-heating, ingot surface may be affected. Hence ingots surface will be favorable region for static recrystallization and grain growth. Many authors agreed over deformations/strains and temperature as the controlling parameters in grain growth. Figure 11 and 12 support the previous line statement as recrystallization can occur during forging or reheating process. On top surface the failure was due to the abnormal grain growth and at base of the crack chromium amount was the reason of failure due to precipitation. But there is another prospect of the failure which was the furnace holding time. It means how long the ingot remained in the furnace for the various HSLA processes. If longer it stay more will be the abnormal growth of grain in later stages of forging and reheating. Barella said even ingot stayed for just 4 hours extra it will impart accountable change in grain property.

(Bitterlin et al., 2016)

\section{Conclusions}

Large size ingots develop crack during open die forging and reheating processes which caused cracks. Case study is done to investigate cause of the cracking. For which Characterization techniques i.e. Scanning Electron Microscope (SEM), Energy Dispersion Spectroscopy (EDS) was used in order to know the microstructure and composition of the specimen. Followings are some important conclusions achieved after reviewing experimental results with the help of literature: 
1. Abnormal grain Growth on surface was due to followings reasons:

Longer Holding time during normalizing heating processes which later causing Abnormal Grain Growth during forging process.

In cogging step, there was insufficient strain deformations to induce recrystallization which later leads to the abnormal grain growth in next stages.

2. Excess Chromium content as chromium oxides were present at grain boundary even there was normal grains particles present. This was a clear indication, cracking at that location was caused due to chromium oxide not grains.

Overall, two mechanism dominated to cause cracking in large ingots. Abnormal Grain Growth caused cracking at the surface where there was no signs of chromium oxides whereas chromium oxides caused cracking at the crack base because grains were in normal size at the base locations. Another main or root cause was also identified. It was the holding time of ingots in furnace during the lateral normalizing process, which in later stages caused abnormal grain and cracking curing forging and reheating process. Hence if control furnace holding time, use of standard chromium content and standard temperature heating, this cracking can be avoided.

\section{References}

1. A QABAN, D. O. M. E. A. A., UNIVERSITY OF LONDON, UK. 2019. Review on Hot and Control Rolling of HSLA Steel. Iris Publisher, 3

2. AISI. 2020. Steel Flowlines [Online]. American Iron and Steel Institute Available:

a. https://www.steel.org/steel-technology/ [Accessed].

3. BHADESHIA, H. \& HONEYCOMBE, R. 2017. Steels: microstructure and properties, Butterworth-Heinemann.

4. BICKFORD, J. 1998. Handbook of bolts and bolted joints, CRC press.

5. BITTERLIN, M., LOUCIF, A., CHARBONNIER, N., JAHAZI, M., LAPIERREBOIRE, L.-P. \& MORIN, J.-B. J. E. F. A. 2016. Cracking mechanisms in large size ingots of high nickel content low alloyed steel. 68, 122-131.

6. KONSTRUKCIJSKA, V. M. H. J. M. I. T. 2011. High-strength low-alloy (HSLA) steels. 45, 295-301.

7. LLEWELLYN, D. \& HUDD, R. 1998. Steels: metallurgy and applications, Elsevier.

8. LONG, K. \& WODARSKI, J. S. J. J. O. E.-B. S. W. 2010. The importance of education, understanding, and empirical research in social work: The nuts and bolts of the business. 7, 173-199. 


\section{CASE STUDY | INVESTIGATION OF LARGE SIZE INGOTS CRACKING FAILURE}

9. MANDAL, S. 2015. Steel metallurgy: properties, specifications and applications, McGraw-Hill Education.

10. MANOHAR, P. 1997. Grain growth and continuous cooling transformation behaviour of austenite in Ti-Nb-Mn-Mo microalloyed steels.

11. MATTES, V. R. 1990. Microstructure and mechanical properties of HSLA-100 steel. NAVAL POSTGRADUATE SCHOOL MONTEREY CA.

12. SALLEZ, N. 2014. Recrystallization, abnormal grain growth and ultrafine microstructure of ODS ferritic steels. Université de Grenoble.

13. SHIRDEL, M., MIRZADEH, H. \& PARSA, M. J. M. C. 2014. Abnormal grain growth in AISI 304L stainless steel. 97, 11-17.

14. TOTTEN, G. E. 2006. Steel heat treatment: metallurgy and technologies, CRC press.

15. TOTTEN, G. E., FUNATANI, K. \& XIE, L. 2004. Handbook of metallurgical process design, CRC press.

16. WANG, F. 2017. Austenite grain growth behaviour of HSLA steel during reheating treatment.

a. University of Birmingham. 